\title{
On the Spectrum of the Local $\mathbb{P}^{2}$ Mirror Curve
}

\author{
Rinat Kashaevio and Sergey Sergeev
}

\begin{abstract}
We address the spectral problem of the formally normal quantum mechanical operator associated with the quantised mirror curve of the toric (almost) del Pezzo Calabi-Yau threefold called local $\mathbb{P}^{2}$ in the case of complex values of Planck's constant. We show that the problem can be approached in terms of the Bethe ansatz-type highly transcendental equations.
\end{abstract}

Mathematics Subject Classification. Primary 39A13, Secondary 33E30.

\section{Introduction}

The recent progress in topological string theory reveals connections between spectral theory, integrable systems and local mirror symmetry. The results on linkings of some quantum mechanical spectral problems with integrable systems and conformal field theory $[4,6]$, together with the relation of topological strings in toric Calabi-Yau manifolds to integrable systems $[1,2,10$ $13,17,19,20]$, have lead to the conjecture on the topological string/spectral theory (TS/ST) correspondence $[5,8]$. In many cases, quantisation of mirror curves produces trace class quantum mechanical operators, and according to the TS/ST correspondence, their spectra seem to contain a great deal of information of the enumerative geometry of the underlying Calabi-Yau manifold; see [16] for a review and references therein.

In the case of toric (almost) del Pezzo Calabi-Yau threefold known as local $\mathbb{P}^{2}$, the corresponding operator is of the form

The work is partially supported by Australian Research Council and Swiss National Science Foundation. 


$$
\boldsymbol{O}_{\mathbb{P}^{2}}=\boldsymbol{u}+\boldsymbol{v}+e^{i \hbar / 2} \boldsymbol{v}^{-1} \boldsymbol{u}^{-1}
$$

with invertible positive self-adjoint operators $\boldsymbol{u}$ and $\boldsymbol{v}$ such that

$$
\boldsymbol{u} \boldsymbol{v}=e^{i \hbar} \boldsymbol{v} \boldsymbol{u}
$$

where $\hbar$ is a real strictly positive parameter. With various levels of generality, the spectral problem for similar operators has been addressed in [15] from the perspective of exact WKB approximation, in $[9,18]$ using a matrix integral representation of the eigenfunctions and in $[3,14,22]$ from the standpoint of quantum integrable systems.

In this paper, following the approach of [14,22], we consider the so-called strongly coupled regime for the operator (1) with invertible normal operators $\boldsymbol{u}$ and $\boldsymbol{v}$ satisfying the Heisenberg-Weyl relation (2) where

$$
\left.\hbar=2 \pi e^{i 2 \theta}, \quad \theta \in\right] 0, \pi / 2[.
$$

In this case, we show that a realisation of the operator (1) in the Hilbert space $L^{2}(\mathbb{R})$ is formally normal so that its spectral problem is still well defined provided the operator admits a normal extension. Our main result is Theorem 2 which describes a set of solutions of our spectral problem in terms of generalised the Bethe ansatz-type transcendental equations.

\section{Outline}

In Sect. 2, we introduce the setup, fix notation and conventions and introduce some objects and constructions that will be used subsequently in the rest of the paper. In Sect. 3, we specify our Hamiltonian as an unbounded operator in $L^{2}(\mathbb{R})$, show that it is formally normal, rewrite the corresponding spectral problem as a pair of functional difference equations and discuss a symmetry of the system called F-duality which corresponds to Faddeev's modularity in integrable systems. In Sect. 4, we describe the possible F-dual asymptotics of solutions of the problem. In Sect. 5, we describe formal power series solutions for each asymptotic behaviour at infinity and establish their convergence properties. In Sect. 6, we develop an analytic tool for the realisation of the formal power series solutions with zero radius of convergence as asymptotic expansions of analytic solutions. This tool is based on the important technical Theorem 1. Section 7 is devoted to explicit constructions of analytic solutions. The specific vector spaces of analytic functions on the punctured complex plane $\mathbb{C}_{\neq 0}$ specified by linear difference equations play the central role here. In Sect. 8, we formulate and prove Theorem 2, the main result of the paper.

\section{Definitions and Notation}

\subsection{Heisenberg Operators}

Let $\boldsymbol{x}$ and $\boldsymbol{p}$ be normalised self-adjoint quantum mechanical Heisenberg operators defined by their realisation in the "position representation"

$$
\langle x| \boldsymbol{x}=x\langle x|, \quad\langle x| \boldsymbol{p}=\frac{1}{2 \pi i} \frac{\partial}{\partial x}\langle x|
$$


in the complex Hilbert space $L^{2}(\mathbb{R})$ of square integrable functions with respect to the standard Lebesgue measure. Here, we use Dirac's bra-ket notation so that for any $\psi \in L^{2}(\mathbb{R})$ we write $\psi(x)=\langle x \mid \psi\rangle$,

$$
\langle x|\boldsymbol{x}| \psi\rangle=x\langle x \mid \psi\rangle
$$

if $\psi$ is in the domain of $\boldsymbol{x}$, and

$$
\langle x|\boldsymbol{p}| \psi\rangle=\frac{1}{2 \pi i} \frac{\partial}{\partial x}\langle x \mid \psi\rangle
$$

if $\psi$ is in the domain of $\boldsymbol{p}$. One can easily verify the Heisenberg commutation relation

$$
[\boldsymbol{p}, \boldsymbol{x}]:=\boldsymbol{p} \boldsymbol{x}-\boldsymbol{x} \boldsymbol{p}=\frac{1}{2 \pi i},
$$

where in the right-hand side the scalar is assumed to be the multiple of the identity operator.

\subsection{Heisenberg-Weyl Operators}

We fix $\theta \in] 0, \pi / 2[$, denote

$$
\mathrm{b}:=e^{i \theta}, \overline{\mathrm{b}}:=\frac{1}{\mathrm{~b}}, \mathrm{q}:=e^{\pi i \mathrm{~b}^{2}}, \overline{\mathrm{q}}:=e^{-\pi i \overline{\mathrm{b}}^{2}}, c_{\mathrm{b}}:=\frac{i}{2}(\mathrm{~b}+\overline{\mathrm{b}})=i \cos \theta
$$

and define the normal Heisenberg-Weyl operators

$$
\boldsymbol{u}:=e^{2 \pi \mathrm{b} \boldsymbol{x}}, \quad \boldsymbol{v}:=e^{2 \pi \mathrm{b} p}
$$

together with their Hermitian conjugates

$$
\boldsymbol{u}^{\dagger}=e^{2 \pi \overline{\mathrm{b}} \boldsymbol{x}}, \quad \boldsymbol{v}^{\dagger}=e^{2 \pi \overline{\mathrm{b}} \boldsymbol{p}} .
$$

These operators satisfy (formally) the commutation relations

$$
\boldsymbol{u} \boldsymbol{v}=q^{2} \boldsymbol{v} \boldsymbol{u}, \quad \boldsymbol{u} \boldsymbol{v}^{\dagger}=\boldsymbol{v}^{\dagger} \boldsymbol{u}
$$

so that if $\boldsymbol{a}$ and $\boldsymbol{b}$ are arbitrary elements of the algebra generated by $\boldsymbol{u}$ and $\boldsymbol{v}$, then formally one has $\boldsymbol{a} \boldsymbol{b}^{\dagger}=\boldsymbol{b}^{\dagger} \boldsymbol{a}$.

\subsection{A Sequence of Polynomials}

To any $n \in \mathbb{Z}_{\geq 0}$, we associate a polynomial $P_{n}=P_{n}(q, E) \in \mathbb{Z}\left[q, q^{-1}\right][E]$ of degree $n$ in $E$ defined by the following recurrence equation

$$
P_{n+1}=E P_{n}+\left(q^{n}-q^{-n}\right)\left(q^{n-1}-q^{1-n}\right) P_{n-2}, \quad P_{0}=1 .
$$

Notice the symmetry $P_{n}(q, E)=P_{n}(1 / q, E)$. Denoting $q_{n}:=q^{n}-q^{-n}$, the few first polynomials read as follows:

$$
\begin{aligned}
& P_{0}=1, \quad P_{1}=E, \quad P_{2}=E^{2}, \quad P_{3}=E^{3}+q_{1} q_{2}, \quad P_{4}=E^{4}+\frac{q_{2}^{3}}{q_{1}} E, \\
& P_{5}=E^{5}+\frac{\left(q_{1}^{2}+q_{3}^{2}\right) q_{2}}{q_{1}} E^{2}, \quad P_{6}=E^{6}+\frac{\left(q_{2}^{2}+5\right) q_{2}^{3}}{q_{1}} E^{3}+q_{1} q_{2} q_{4} q_{5},
\end{aligned}
$$

Among the properties of these polynomials, one can show that $P_{n}(q, 0)=0$ unless $n \equiv 0(\bmod 3)$ and

$$
P_{3 m}(q, 0)=q^{-3 m^{2}}\left(q^{2} ; q^{6}\right)_{m}\left(q^{4} ; q^{6}\right)_{m}, \quad \forall m \in \mathbb{Z}_{\geq 0},
$$


where we use the standard $q$-Pochhammer symbol

$$
(x ; q)_{n}:=\prod_{k=0}^{n-1}\left(1-x q^{k}\right), \quad(x, y, \ldots ; q)_{n}:=(x ; q)_{n}(y ; q)_{n} \cdots
$$

One can also show that

$$
P_{n}=\sum_{0 \leq m \leq n / 3} E^{n-3 m} p_{n, m}, \quad p_{n, m}=\sum_{|k| \leq m} q^{2 n k} \alpha_{k, m}(n),
$$

where $\alpha_{k, m}(x)$ are polynomials in $x$ of degree $m-|k|$ satisfying the recurrence relations

$$
\begin{aligned}
& q^{6 k} \alpha_{k, m+1}(x+3)-q^{4 k} \alpha_{k, m+1}(x+2) \\
& \quad=q^{3} \alpha_{k-1, m}(x)+q^{-3} \alpha_{k+1, m}(x)-\left(q+q^{-1}\right) \alpha_{k, m}(x) .
\end{aligned}
$$

Furthermore, the leading asymptotics of $P_{n}$ at large $n$ is given by the formula

$$
\left.P_{n}(q, E)\right|_{n \rightarrow \infty} \sim q^{-n^{2} / 3} .
$$

It will be of particular interest for us the following two generating series for these polynomials:

$$
\phi_{q, E}(z):=\sum_{n=0}^{\infty} \frac{P_{n}(q, E)}{\left(q^{-2} ; q^{-2}\right)_{n}} z^{n}
$$

and

$$
\psi_{q, E}(z):=\sum_{n=0}^{\infty} \frac{P_{n}(q, E) q^{(1-n) n / 2}}{\left(q^{-2} ; q^{-2}\right)_{n}} z^{n}=\psi_{1 / q, E}(-z) .
$$

The asymptotics (18) implies that for any $E \in \mathbb{C}$, the radius of convergence is infinite for the series (20), while for the series (19) it is infinite if $|q|<1$ and zero if $|q|>1$.

\subsection{Vector Spaces $F_{p, c}, G_{p, c}$ and $T_{p, r}^{m}$}

We let $H\left(\mathbb{C}_{\neq 0}\right)$ denote the complex vector space of holomorphic functions $f: \mathbb{C}_{\neq 0} \rightarrow \mathbb{C}$ and

$$
U(f):=\mathbb{C}_{\neq 0} \backslash f^{-1}(0), \quad f \in H\left(\mathbb{C}_{\neq 0}\right) .
$$

Let $c \in \mathbb{C}, p, r \in \mathbb{C}_{\neq 0}$ and $m \in \mathbb{Z}$. We define the following vector subspaces of $H\left(\mathbb{C}_{\neq 0}\right)$ :

$$
\begin{aligned}
& F_{p, c}:=\left\{f \in H\left(\mathbb{C}_{\neq 0}\right) \mid f\left(z / p^{2}\right)+(z p)^{3} f\left(z p^{2}\right)=(1-c z) f(z)\right\}, \\
& G_{p, c}:=\left\{f \in H\left(\mathbb{C}_{\neq 0}\right) \mid f(z p)-f(z / p)=z\left(z^{2}+c\right) f(z)\right\}
\end{aligned}
$$

and

$$
T_{p, r}^{m}:=\left\{f \in H\left(\mathbb{C}_{\neq 0}\right) \mid r z^{m} f(z p)=f(z)\right\} .
$$

Elements of $T_{p, r}^{m}$ will be called theta functions of order $m$. For any $p \in \mathbb{C}_{\neq 0}$ such that $|p|<1$, one specific theta function is defined by the series

$$
\vartheta(z ; p):=\sum_{n \in \mathbb{Z}} p^{(n-1) n / 2}(-z)^{n}=(z, p / z, p ; p)_{\infty}
$$


with the defining properties

$$
\vartheta(1 ; p)=0, \quad \vartheta(p z ; p)=\vartheta\left(z^{-1} ; p\right)=-\frac{1}{z} \vartheta(z ; p)
$$

which imply that $\vartheta(\cdot ; p) \in T_{p,-1}^{1}$. Note also the modularity property

$$
\vartheta\left(e^{2 \pi \mathrm{b} x} ; \mathrm{q}^{2}\right)=\vartheta\left(e^{2 \pi \overline{\mathrm{b}} x} ; \overline{\mathrm{q}}^{2}\right) e^{\pi i(x+\sin \theta)^{2}-i \theta+\pi i / 4},
$$

where we use notation (8).

Remark 1. Let $f \in T_{p, r}^{m}$ and $z \in U(f)$ (respectively, $\left.z \notin U(f)\right)$. Then, one has $z p^{\mathbb{Z}} \subset U(f)$ (respectively, $z p^{\mathbb{Z}} \cap U(f)=\{\}$ ).

Remark 2. By expanding into Laurent series, one easily checks that the dimensions of $F_{p, c}$ and $G_{p, c}$ are at most 3. On the other hand, the recurrence relation (12) implies that $\psi_{p, c} \in G_{p, c}$ if $|p| \neq 1$ and $\phi_{p, c} \in F_{p, c}$ if $|p|<1$ so that

$$
|p| \neq 1 \Rightarrow \operatorname{dim} G_{p, c} \geq 1
$$

and

$$
|p|<1 \Rightarrow \operatorname{dim} F_{p, c} \geq 1 .
$$

The elements $\phi_{p, c}$ and $\psi_{p, c}$ will be called regular elements of the corresponding vector spaces.

Remark 3. By expanding into Laurent series, it is easily verified that

$$
\operatorname{dim} T_{p, r}^{m} \leq|m| \text { if } m \neq 0
$$

with the equality if $|p|^{m}<1$. In particular, for $|p|<1, \operatorname{dim} T_{p, r}^{1}=1$ with the theta function $\vartheta(-r z ; p)$ being a basis element.

Remark 4. One has the identifications

$$
\begin{aligned}
& T_{1,1}^{0}=H\left(\mathbb{C}_{\neq 0}\right), \\
& T_{p, 1}^{0}=\mathbb{C} \text { if } 1 \notin p^{\mathbb{Z}_{\neq 0}},
\end{aligned}
$$

and the inclusions

$$
T_{p, r}^{m} \subset T_{p^{n}, r^{n} p^{m n(n-1) / 2}}^{m n}, \quad \forall m, n \in \mathbb{Z}, \forall p, r \in \mathbb{C}_{\neq 0},
$$

which for $n=-1$ become equalities

$$
T_{p, r}^{m}=T_{p^{-1}, r^{-1} p^{m}}^{-m}, \quad \forall m \in \mathbb{Z}, \forall p, r \in \mathbb{C}_{\neq 0} .
$$

Remark 5. The multiplication of functions induces a linear map

$$
T_{p, r}^{m} \otimes T_{p, s}^{n} \rightarrow T_{p, r s}^{m+n}, \quad \forall m, n \in \mathbb{Z}, \forall p, r, s \in \mathbb{C}_{\neq 0} .
$$

For example, assuming $|p|<1$, the product identity

$$
\vartheta(z ; p) \vartheta(-z ; p)=\vartheta\left(p ; p^{2}\right) \vartheta\left(z^{2} ; p^{2}\right)
$$

illustrates the special case $T_{p,-1}^{1} \otimes T_{p, 1}^{1} \rightarrow T_{p,-1}^{2}$. 
Remark 6. Assuming $1 \notin p^{\mathbb{Z} \neq 0}$, let $g \in G_{p, c}$. Then, the even part of the product $g(z) g(-z p)$ is an element of the vector space $T_{p, 1}^{0}=\mathbb{C}$. Thus, there exists a quadratic form $\omega: G_{p, c} \rightarrow \mathbb{C}$ such that

$$
g(z) g(-z p)+g(-z) g(z p)=\omega(g), \quad \forall z \in \mathbb{C}_{\neq 0} .
$$

In particular, if $|p| \neq 1$, we have

$$
\omega\left(\psi_{p, c}\right)=2 \psi_{p, c}(0)=2 .
$$

\section{Formulation of the Spectral Problem}

Let $\boldsymbol{u}$ and $\boldsymbol{v}$ be the normal Heisenberg-Weyl operators defined in (9). Then, the Hamiltonian

$$
\begin{aligned}
\boldsymbol{H} & :=\boldsymbol{u}+\boldsymbol{v}+\mathrm{q}^{-1} \boldsymbol{u}^{-1} \boldsymbol{v}^{-1}=\boldsymbol{u}+\boldsymbol{v}+\mathrm{q} \boldsymbol{v}^{-1} \boldsymbol{u}^{-1} \\
& =e^{2 \pi \mathrm{b} \boldsymbol{x}}+e^{2 \pi \mathrm{b} \boldsymbol{p}}+e^{-2 \pi \mathrm{b}(\boldsymbol{x}+\boldsymbol{p})}
\end{aligned}
$$

with the standard domain for sums of operators

$$
\mathcal{D}(\boldsymbol{H})=\mathcal{D}\left(e^{2 \pi \mathrm{b} \boldsymbol{x}}\right) \cap \mathcal{D}\left(e^{2 \pi \mathrm{b} \boldsymbol{p}}\right) \cap \mathcal{D}\left(e^{-2 \pi \mathrm{b}(\boldsymbol{x}+\boldsymbol{p})}\right)
$$

is expected to extend to a normal operator so that the spectral problem consisting in solving the system of Schrödinger equations

$$
\boldsymbol{H}|\Psi\rangle=E|\Psi\rangle, \quad \boldsymbol{H}^{\dagger}|\Psi\rangle=\bar{E}|\Psi\rangle
$$

in the Hilbert space $L^{2}(\mathbb{R})$ is well posed. Below we justify this expectation by showing that $\boldsymbol{H}$ is at least formally normal.

\subsection{The formal Normality of $\boldsymbol{H}$}

Recall that a densely defined linear operator $T$ with the domain $\mathcal{D}(T)$ is called formally normal if $\mathcal{D}(T) \subset \mathcal{D}\left(T^{\dagger}\right)$ and

$$
\|T x\|=\left\|T^{\dagger} x\right\| \quad \forall x \in \mathcal{D}(T) .
$$

A formally normal operator $T$ is called normal if $\mathcal{D}(T)=\mathcal{D}\left(T^{\dagger}\right)$; see, for example, [21]. The notion of a formally normal operator naturally generalises the notion of a symmetric operator when one extends the class of self-adjoint operators to the class of normal operators.

Lemma 1. Let $\left\{\boldsymbol{a}_{j}\right\}_{j \in J}$ be a finite set of densely defined operators such that $\boldsymbol{A}:=\sum_{j \in J} \boldsymbol{a}_{j}$ is densely defined, and for any $j, k \in J$, the operator $\boldsymbol{a}_{j}+\boldsymbol{a}_{k}$ is formally normal. Then $\boldsymbol{A}$ is formally normal as well.

Proof. The case with $j=k$ implies that the operator $\boldsymbol{a}_{j}$ is formally normal for any $j \in J$. This immediately implies the inclusion of domains

$$
\mathcal{D}(\boldsymbol{A})=\bigcap_{j \in J} \mathcal{D}\left(\boldsymbol{a}_{j}\right) \subset \bigcap_{j \in J} \mathcal{D}\left(\boldsymbol{a}_{j}^{\dagger}\right)=\mathcal{D}\left(\sum_{j \in J} \boldsymbol{a}_{j}^{\dagger}\right) \subset \mathcal{D}\left(\boldsymbol{A}^{\dagger}\right),
$$

where the last inclusion is due to the inclusion of operators ${ }^{1} \sum_{j \in J} \boldsymbol{a}_{j}^{\dagger} \subset \boldsymbol{A}^{\dagger}$.

\footnotetext{
${ }^{1}$ By inclusion of operators $S \subset T$, we mean that $\mathcal{D}(S) \subset \mathcal{D}(T)$ and $S=\left.T\right|_{\mathcal{D}(S)}$.
} 
For any $j, k \in J$, by taking into account the inclusion of operators $\boldsymbol{a}_{j}^{\dagger}+$ $\boldsymbol{a}_{k}^{\dagger} \subset\left(\boldsymbol{a}_{j}+\boldsymbol{a}_{k}\right)^{\dagger}$ as well as the formal normality of $\boldsymbol{a}_{j}$ and $\boldsymbol{a}_{k}$, we have the following equivalent characterisation of the formal normality of $\boldsymbol{a}_{j}+\boldsymbol{a}_{k}$ :

$$
M_{j, k}(x)=-M_{k, j}(x) \quad \forall x \in \mathcal{D}\left(\boldsymbol{a}_{j}+\boldsymbol{a}_{k}\right),
$$

where

$$
M_{j, k}(x):=\left\langle\boldsymbol{a}_{j} x \mid \boldsymbol{a}_{k} x\right\rangle-\left\langle\boldsymbol{a}_{j}^{\dagger} x \mid \boldsymbol{a}_{k}^{\dagger} x\right\rangle .
$$

Indeed, this is verified as follows:

$$
\begin{aligned}
0 & =\left\|\left(\boldsymbol{a}_{j}+\boldsymbol{a}_{k}\right) x\right\|^{2}-\left\|\left(\boldsymbol{a}_{j}+\boldsymbol{a}_{k}\right)^{\dagger} x\right\|^{2}=\left\|\left(\boldsymbol{a}_{j}+\boldsymbol{a}_{k}\right) x\right\|^{2}-\left\|\left(\boldsymbol{a}_{j}^{\dagger}+\boldsymbol{a}_{k}^{\dagger}\right) x\right\|^{2} \\
& =M_{j, k}(x)+M_{k, j}(x) .
\end{aligned}
$$

Thus, for any $x \in \mathcal{D}(\boldsymbol{A})$, we obtain

$$
\begin{aligned}
\|\boldsymbol{A} x\|^{2}- & \left\|\boldsymbol{A}^{\dagger} x\right\|^{2}=\sum_{j, k \in J} M_{j, k}(x)=-\sum_{j, k \in J} M_{k, j}(x)=\left\|\boldsymbol{A}^{\dagger} x\right\|^{2}-\|\boldsymbol{A} x\|^{2} \\
\Leftrightarrow\|\boldsymbol{A} x\| & =\left\|\boldsymbol{A}^{\dagger} x\right\| .
\end{aligned}
$$

Lemma 2. Let $\mathrm{b}=e^{i \theta}$ with $\left.\theta \in\right] 0, \frac{\pi}{2}[$. Then, the set of three operators

$$
\left\{\boldsymbol{a}_{1}:=e^{2 \pi \mathrm{b} \boldsymbol{x}}, \boldsymbol{a}_{2}:=e^{2 \pi \mathrm{b} \boldsymbol{p}}, \boldsymbol{a}_{3}:=e^{-2 \pi \mathrm{b}(\boldsymbol{x}+\boldsymbol{p})}\right\}
$$

satisfies the assumptions of Lemma 1.

Proof. For any $j \in\{1,2,3\}$, the operator $\boldsymbol{a}_{j}$ is obviously normal. Let us check that $\boldsymbol{a}_{1}+\boldsymbol{a}_{2}$ is formally normal.

By using notation (8), recall that Faddeev's quantum dilogarithm

$$
\Phi_{\mathrm{b}}(z)=\frac{\left(-\mathrm{q} e^{2 \pi \mathrm{b} z} ; \mathrm{q}^{2}\right)_{\infty}}{\left(-\overline{\mathrm{q}} e^{2 \pi \overline{\mathrm{b}} z} ; \overline{\mathrm{q}}^{2}\right)_{\infty}}
$$

is a meromorphic function of $z \in \mathbb{C}$ which satisfies the functional equation

$$
\frac{\Phi_{\mathrm{b}}(z-i \mathrm{~b} / 2)}{\Phi_{\mathrm{b}}(z+i \mathrm{~b} / 2)}=1+e^{2 \pi \mathrm{b} z}
$$

and the unitarity condition

$$
\overline{\Phi_{\mathrm{b}}(z)} \Phi_{\mathrm{b}}(\bar{z})=1,
$$

and it has neither zeros nor poles in the strip $|\Im(z)|<\cos (\theta)$. By using these properties, we write

$$
\begin{aligned}
\boldsymbol{a}_{1}+\boldsymbol{a}_{2} & =e^{2 \pi \mathrm{b} \boldsymbol{x}}+e^{2 \pi \mathrm{b} \boldsymbol{p}}=e^{\pi \mathrm{b} \boldsymbol{x}}\left(1+e^{2 \pi \mathrm{b}(\boldsymbol{p}-\boldsymbol{x})}\right) e^{\pi \mathrm{b} \boldsymbol{x}} \\
& =e^{\pi \mathrm{b} \boldsymbol{x}} \frac{\Phi_{\mathrm{b}}(\boldsymbol{p}-\boldsymbol{x}-i \mathrm{~b} / 2)}{\Phi_{\mathrm{b}}(\boldsymbol{p}-\boldsymbol{x}+i \mathrm{~b} / 2)} e^{\pi \mathrm{b} \boldsymbol{x}}=U e^{2 \pi \mathrm{b} x} U^{\dagger},
\end{aligned}
$$

where $U:=\Phi_{\mathrm{b}}(\boldsymbol{p}-\boldsymbol{x})$ is a unitary operator and we used the following equalities:

$$
e^{\pi s \boldsymbol{x}} \Phi_{\mathrm{b}}(\boldsymbol{p}-\boldsymbol{x}-i s / 2)=\Phi_{\mathrm{b}}(\boldsymbol{p}-\boldsymbol{x}) e^{\pi s \boldsymbol{x}}=U e^{\pi s \boldsymbol{x}}, \quad s \in\{\mathrm{b}, \overline{\mathrm{b}}\}
$$


which hold true due to the fact that the complex shifts in the argument of $\Phi_{\mathrm{b}}(z)$ are within the strip free of zeros and poles:

$$
|\Im(i \mathrm{~b} / 2)|=|\Im(i \overline{\mathrm{b}} / 2)|=\frac{1}{2} \cos (\theta)<\cos (\theta) .
$$

Thus, $\boldsymbol{a}_{1}+\boldsymbol{a}_{2}$ admits a normal extension, and in particular, it is formally normal. The formal normality of $\boldsymbol{a}_{1}+\boldsymbol{a}_{3}$ and $\boldsymbol{a}_{2}+\boldsymbol{a}_{3}$ is verified similarly.

We conclude that Lemma 1 in conjuction with Lemma 2 implies that the Hamiltonian (39) is a formally normal operator.

\subsection{The Difference Equations and F-Duality}

In the position representation (4), the spectral problem (41) is equivalent to the following system of functional difference equations

$$
\begin{aligned}
\Psi(x-i \mathrm{~b})+\mathrm{q}^{-1} e^{-2 \pi \mathrm{b} x} \Psi(x+i \mathrm{~b}) & =\left(E-e^{2 \pi \mathrm{b} x}\right) \Psi(x), \\
\Psi(x-i \overline{\mathrm{b}})+\overline{\mathrm{q}} e^{-2 \pi \overline{\mathrm{b}} x} \Psi(x+i \overline{\mathrm{b}}) & =\left(\bar{E}-e^{2 \pi \overline{\mathrm{b}} x}\right) \Psi(x),
\end{aligned}
$$

where $\Psi(x):=\langle x \mid \Psi\rangle$. We are looking for a holomorphic function $\Psi: \mathbb{C} \rightarrow \mathbb{C}$ that solves the functional Eqs. (55), (56) and whose restriction to the real axis is square integrable.

Equations (55) and (56) are related to each other by the simultaneous substitutions

$$
\mathrm{b} \leftrightarrow \overline{\mathrm{b}}, \quad \mathrm{q} \leftrightarrow 1 / \overline{\mathrm{q}}, \quad E \leftrightarrow \bar{E}
$$

which correspond to the Faddeev (modular) duality [7] and which we abbreviate as F-duality. For this reason, in what follows, we will write only one equation (containing the variables $E$ and $\mathrm{q}$ ), but implicitly there will always be a second accompanying equation. In constructing solutions, we will follow the principle of F-duality corresponding to the invariance of solutions under above substitutions. In this case, it will suffice to check only one equation as the other one will be satisfied automatically.

\section{F-dual Asymptotics at $x \rightarrow \pm \infty$}

We start our analysis by addressing the problem of asymptotical behaviour of solutions of our spectral problem at large values of $x$. Following the principle of F-duality, we are looking for possible F-dual asymptotics.

Proposition 1. Let $\Psi(x)$ be a solution of Eqs. (55) and (56) such that there exists a finite nonzero limit of $e^{\lambda x} \Psi(x-i \mathrm{~b}) / \Psi(x)$ when $x$ tends either to $+\infty$ or to $-\infty$ for some $\lambda \in \mathbb{C}$. Then, one has the following possibilities for the $F$-dual asymptotic behaviour of $\Psi(x)$ at large $x$ :

$$
\begin{aligned}
& \left.\Psi(x)\right|_{x \rightarrow-\infty} \sim \psi_{0}(x):=e^{-\pi i x^{2} / 2-\pi i c_{\mathrm{b}} x}, \\
& \left.\Psi(x)\right|_{x \rightarrow+\infty} \sim \psi_{1}(x):=e^{\pi i x^{2}+2 \pi i c_{\mathrm{b}} x},
\end{aligned}
$$

and

$$
\left.\Psi(x)\right|_{x \rightarrow+\infty} \sim \psi_{2}(x):=e^{-2 \pi i x^{2}+2 \pi i c_{\mathrm{b}} x} .
$$


Proof. Dividing (55) by $\Psi(x)$, and denoting $\rho_{\lambda}(x):=e^{\lambda x} \Psi(x-i \mathrm{~b}) / \Psi(x)$, we obtain a first-order nonlinear finite difference functional equation with exponentially growing or decaying coefficients

$$
\rho_{\lambda}(x)+e^{(2 x+i \mathbf{b})(\lambda-\pi \mathbf{b})} / \rho_{\lambda}(x+i \mathbf{b})=\left(E-e^{2 \pi \mathbf{b} x}\right) e^{x \lambda} .
$$

Let $\lambda \in \mathbb{C}$ be such that there exists a finite nonzero limit value

$$
\mu(\lambda):=\lim _{x \rightarrow \infty} \rho_{\lambda}(x)=\lim _{x \rightarrow \infty} \rho_{\lambda}(x+i \mathrm{~b}),
$$

where $\infty$ means one of $\pm \infty$, and assume that there exists an F-dual solution of the finite difference functional equation

$$
e^{\lambda x} f_{\lambda}(x-i \mathrm{~b}) / f_{\lambda}(x)=\mu(\lambda)
$$

Then, the corresponding asymptotic behaviour of $\Psi(x)$ is given by $f_{\lambda}(x)$.

The case $x \rightarrow-\infty$. Choosing $\lambda=\pi \mathrm{b}$, we obtain

$$
\lim _{x \rightarrow-\infty}\left(\rho_{\pi \mathrm{b}}(x)+\frac{1}{\rho_{\pi \mathrm{b}}(x+i \mathrm{~b})}\right)=0 .
$$

Thus, one has two possibilities for the limit value

$$
\mu(\pi \mathrm{b})=\epsilon i, \quad \epsilon \in\{ \pm 1\} .
$$

The finite difference functional equation (63) admits an F-dual solution of the form $f_{\pi \mathrm{b}}(x)=\psi_{0}(x)$ provided $\epsilon=-1$.

The case $x \rightarrow+\infty$. We can choose either $\lambda=-2 \pi \mathrm{b}$ or $\lambda=4 \pi \mathrm{b}$ with the limit values

$$
\mu(-2 \pi \mathrm{b})=-1, \quad \mu(4 \pi \mathrm{b})=-\mathrm{q}^{3} .
$$

The corresponding F-dual solutions of (63) are given by $f_{-2 \pi \mathrm{b}}(x)=\psi_{1}(x)$ and $f_{4 \pi \mathrm{b}}(x)=\psi_{2}(x)$.

Our further goal is to try to solve the eigenvalue Eqs. (55) and (56) in three asymptotic regimes (58)-(60) by using the substitutions $\Psi(x)=$ $\psi_{i}(x) \varphi_{i}(x), i \in\{0,1,2\}$ and looking for functions $\varphi_{i}(x)$ having finite nonzero limiting values in the corresponding asymptotic limits.

\section{Solutions in Terms of Power Series}

\subsection{The Case $\psi_{2}(x)$}

Assume that the asymptotic behaviour (60) takes place. If we write $\Psi(x)=$ $\psi_{2}(x) \varphi_{2}(x)$, then the eigenvalue equation (55) is converted into the equation

$$
\varphi_{2}(x+i \mathrm{~b})+e^{-6 \pi \mathrm{b} x} \mathrm{q}^{3} \varphi_{2}(x-i \mathrm{~b})=\left(1-E e^{-2 \pi \mathrm{b} x}\right) \varphi_{2}(x)
$$

which we complement with the limit value condition

$$
\lim _{x \rightarrow+\infty} \varphi_{2}(x)=1 .
$$

Under the F-dual substitution

$$
\varphi_{2}(x)=\chi_{2}\left(e^{2 \pi \mathrm{b} x}\right) \bar{\chi}_{2}\left(e^{2 \pi \overline{\mathrm{b}} x}\right),
$$


(67) is reduced to the equation

$$
\chi_{2}\left(z \mathrm{q}^{2}\right)+(\mathrm{q} / z)^{3} \chi_{2}\left(z / \mathrm{q}^{2}\right)=(1-E / z) \chi_{2}(z)
$$

which we complement with the limit value condition

$$
\lim _{z \rightarrow \infty} \chi_{2}(z)=1 \text {. }
$$

It admits a power series solution

$$
\chi_{2}(z)=\phi_{\mathrm{q}, E}(1 / z) .
$$

The dual function $\bar{\chi}_{2}(z)$ is obtained by the substitutions $\mathrm{q} \mapsto 1 / \overline{\mathrm{q}}$ and $E \mapsto \bar{E}$ with the result

$$
\bar{\chi}_{2}(z)=\phi_{1 / \bar{q}, \bar{E}}(1 / z) .
$$

Taking into account the remarks in the end of Sect. 2.3, we conclude that $\chi_{2}(z)$ is holomorphic in $\mathbb{C}_{\neq 0}$, while $\bar{\chi}_{2}(z)$ does not converge to any complex analytic function.

\subsection{The Case $\psi_{1}(x)$}

Assume that the behaviour (59) takes place. If we write $\Psi(x)=\psi_{1}(x) \varphi_{1}(x)$, then the eigenvalue equation (55) is converted into the equation

$$
\varphi_{1}(x-i \mathrm{~b})+e^{-6 \pi \mathrm{b} x} \mathrm{q}^{-3} \varphi_{1}(x+i \mathrm{~b})=\left(1-E e^{-2 \pi \mathrm{b} x}\right) \varphi_{1}(x)
$$

which we complement with the limit value condition

$$
\lim _{x \rightarrow+\infty} \varphi_{1}(x)=1 .
$$

As in the previous case, we obtain a power series F-dual solution

$$
\varphi_{1}(x)=\phi_{1 / \mathrm{q}, E}\left(e^{-2 \pi \mathrm{b} x}\right) \phi_{\overline{\mathrm{q}}, \bar{E}}\left(e^{-2 \pi \overline{\mathrm{b}} x}\right),
$$

where the radii of convergence of the series $\phi_{1 / \mathrm{q}, E}(z)$ and $\phi_{\overline{\mathrm{q}}, \bar{E}}(z)$ are zero and infinity, respectively.

\subsection{The Case $\psi_{0}(x)$}

The substitution $\Psi(x)=\psi_{0}(x) \varphi_{0}(x)$ converts the eigenvalue equation (55) into the equation

$$
\varphi_{0}(x-i \mathrm{~b})-\varphi_{0}(x+i \mathrm{~b})=i\left(E-e^{2 \pi \mathrm{b} x}\right) e^{\pi \mathrm{b} x} \varphi_{0}(x)
$$

which we complement with the limit value condition

$$
\lim _{x \rightarrow-\infty} \varphi_{0}(x)=1 .
$$

Under the F-dual substitution

$$
\varphi_{0}(x)=\frac{1}{2} \sum_{\sigma, \tau \in\{0,1\}}(-1)^{\sigma \tau} \chi_{0}\left((-1)^{\sigma} e^{\pi \mathrm{b} x}\right) \bar{\chi}_{0}\left((-1)^{\tau} e^{\pi \overline{\mathrm{b}} x}\right),
$$

(77) is equivalent to the following functional equation on the function $\chi_{0}(z)$ :

$$
\chi_{0}(z / \mathbf{q})-\chi_{0}(z \mathbf{q})=i\left(E-z^{2}\right) z \chi_{0}(z)
$$

complemented with the initial value condition

$$
\chi_{0}(0)=1 \text {. }
$$


It admits a power series solution $\chi_{0}(z)=\psi_{\mathbf{q}, E}(-i z)$ and its dual $\bar{\chi}_{0}(z)=$ $\psi_{\overline{\mathbf{q}}, \bar{E}}(i z)$ with infinite radii of convergence. Thus, in this case, we obtain an entire function $\Psi(x)$.

\section{Analytic Realisations of the Series $\phi_{1 / \mathrm{q}, E}(z)$}

\subsection{First-Order Matrix Difference Equation}

For any $f \in F_{\mathbf{q}, E}$, we have a matrix equality

$$
\hat{f}(z)=L(z) \hat{f}\left(z \mathrm{q}^{2}\right), \quad \hat{f}(z):=\left(\begin{array}{c}
f\left(\frac{z}{\mathrm{q}^{2}}\right) \\
f(z)
\end{array}\right), L(z):=\left(\begin{array}{cc}
1-E z & -z^{3} \mathrm{q}^{3} \\
1 & 0
\end{array}\right) .
$$

Defining

$$
L_{n}(z):=L(z) L\left(z \mathrm{q}^{2}\right) \cdots L\left(z \mathrm{q}^{2 n-2}\right)=:\left(\begin{array}{ll}
a_{n}(z) & b_{n}(z) \\
c_{n}(z) & d_{n}(z)
\end{array}\right), \quad n \in \mathbb{Z}_{>0},
$$

we have

$$
L_{m+n}(z)=L_{m}(z) L_{n}\left(z \mathrm{q}^{2 m}\right), \quad \forall(m, n) \in\left(\mathbb{Z}_{>0}\right)^{2},
$$

which, in particular, implies that

$$
L_{n+1}(z)=L(z) L_{n}\left(z \mathrm{q}^{2}\right)=L_{n}(z) L\left(z \mathrm{q}^{2 n}\right), \quad \forall n \in \mathbb{Z}_{>0} .
$$

Taking the limit $n \rightarrow \infty$ in (83), relations (85) imply that

$$
L_{\infty}(z):=\lim _{n \rightarrow \infty} L_{n}(z)=\left(\begin{array}{cc}
\phi_{\mathbf{q}, E}\left(z / \mathbf{q}^{2}\right) & 0 \\
\phi_{\mathbf{q}, E}(z) & 0
\end{array}\right),
$$

where $\phi_{\mathrm{q}, E}$ is the regular element of $F_{\mathrm{q}, E}$. As we have seen in Sect. 2.3, it can be presented as the everywhere absolutely convergent series (19).

\subsection{The Wronskian Pairing}

We define a skew-symmetric bilinear Wronskian pairing

$$
[\cdot, \cdot]: F_{\mathrm{q}, E} \times F_{\mathrm{q}, E} \rightarrow T_{\mathrm{q}^{2}, \mathrm{q}^{3}}^{3}, \quad[f, g](z)=f\left(\frac{z}{\mathrm{q}^{2}}\right) g(z)-g\left(\frac{z}{\mathrm{q}^{2}}\right) f(z) .
$$

Remark \%. One can show that $\operatorname{dim} F_{\mathrm{q}, E}=3$. As the kernel of the Wronskian pairing $\left[\phi_{\mathrm{q}, E}, \cdot\right]$ contains $\phi_{\mathrm{q}, E}$, we conclude that $\operatorname{dim}\left[\phi_{\mathrm{q}, E}, F_{\mathrm{q}, E}\right] \leq 2$.

\subsection{Adjoint Functions}

For any $f \in F_{\mathbf{q}, E}$, we associate the adjoint function

$$
\tilde{f}: U\left(\left[\phi_{\mathbf{q}, E}, f\right]\right) \rightarrow \mathbb{C}
$$

(see (21) for the definition of the domain) defined by the formula

$$
\tilde{f}(z):=\frac{f(z)}{\left[\phi_{\mathbf{q}, E}, f\right](z)} .
$$

By construction, $\tilde{f}(z)$ solves the functional equation

$$
\tilde{f}\left(z \mathbf{q}^{2}\right)+(z / \mathbf{q})^{3} \tilde{f}\left(z / \mathbf{q}^{2}\right)=(1-E z) \tilde{f}(z)
$$


obtained from the equation underlying the vector space $F_{\mathrm{q}, E}$ by the replacement of q by $1 / \mathrm{q}$. This equation admits a formal power series solution $\phi_{1 / \mathrm{q}, E}(z)$ which has zero radius of convergence. The adjoint functions appear to be analytic substitutes for $\phi_{1 / \mathrm{q}, E}$ due to the following theorem.

Theorem 1. Let $f \in F_{\mathrm{q}, E}$ be such that the adjoint function $\tilde{f}(z)$ is a non-trivial meromorphic function. Then

$$
\lim _{n \rightarrow \infty} \tilde{f}\left(z \mathrm{q}^{2 n}\right)=1, \quad \forall z \in U\left(\left[\phi_{\mathrm{q}, E}, f\right]\right),
$$

so that $\tilde{f}(z)$ admits an asymptotic expansion at small $z$ in the form of the series $\phi_{1 / \mathrm{q}, E}(z)$.

Proof. The proof is based on the matrix recurrence (82). Indeed, the formula

$$
\operatorname{det}\left(L_{n}(z)\right)=z^{3 n} \mathrm{q}^{3 n^{2}}
$$

implies that $L_{n}(z)$ is invertible for any $z \neq 0$, and we can write

$$
\hat{f}(z)=L_{n}(z) \hat{f}\left(z \mathrm{q}^{2 n}\right) \Leftrightarrow \hat{f}\left(z \mathrm{q}^{2 n}\right)=\left(L_{n}(z)\right)^{-1} \hat{f}(z)
$$

so that

$$
f\left(z \mathrm{q}^{2 n}\right)=\frac{a_{n}(z) f(z)-c_{n}(z) f\left(z / \mathrm{q}^{2}\right)}{z^{3 n} \mathrm{q}^{3 n^{2}}},
$$

and taking into account the equality

$$
\left[\phi_{\mathbf{q}, E}, f\right]\left(z \mathbf{q}^{2 n}\right)=\frac{\left[\phi_{\mathbf{q}, E}, f\right](z)}{z^{3 n} \mathbf{q}^{3 n^{2}}},
$$

we obtain

$$
\tilde{f}\left(z \mathrm{q}^{2 n}\right)=\frac{a_{n}(z) f(z)-c_{n}(z) f\left(z / \mathrm{q}^{2}\right)}{\left[\phi_{\mathrm{q}, E}, f\right](z)}
$$

which implies (91) due to the formulae

$$
\lim _{n \rightarrow \infty} a_{n}(z)=\phi_{\mathbf{q}, E}\left(z / \mathrm{q}^{2}\right), \quad \lim _{n \rightarrow \infty} c_{n}(z)=\phi_{\mathbf{q}, E}(z),
$$

see (86), and the definition of the Wronskian pairing in (87).

Our next task is to construct elements of $F_{\mathrm{q}, E}$ with non-trivial adjoint functions.

\section{Construction of Elements in $\boldsymbol{F}_{\mathrm{q}, E}$}

\subsection{The Vector Space $V_{\mathrm{q}, \alpha, E}$}

Let $\alpha \in \mathbb{C}_{\neq 0}$. We define a vector space

$$
V_{\mathrm{q}, \alpha, E}:=\left\{f \in H\left(\mathbb{C}_{\neq 0}\right) \mid \alpha z f\left(\frac{z}{\mathrm{q}^{2}}\right)+\frac{z^{2} \mathrm{q}}{\alpha} f\left(z \mathrm{q}^{2}\right)=(1-E z) f(z)\right\} .
$$


Proposition 2. Let $g \in G_{\mathbf{q}, E}$. Consider the linear map

$$
A_{g}: H\left(\mathbb{C}_{\neq 0}\right) \rightarrow H\left(\mathbb{C}_{\neq 0}\right), \quad A_{g}(f)(z)=P_{+}(f g)(1 / \sqrt{-z}),
$$

where $P_{+}$is the projection to the even part of a function:

$$
P_{+}(f)(z):=(f(z)+f(-z)) / 2 .
$$

Then $A_{g}\left(T_{\mathrm{q},-\alpha}^{1}\right) \subset V_{\mathrm{q}, \alpha, E}$ and the restriction $\left.A_{g}\right|_{T_{\mathrm{q},-\alpha}^{1}}$ is a linear isomorphism between $T_{\mathbf{q},-\alpha}^{1}$ and $V_{\mathbf{q}, \alpha, E}$ provided $\omega(g) \neq 0$ (see Remark 6 ).

Proof. Let $h \in T_{\mathrm{q},-\alpha}^{1}$ and $f:=A_{g}(h)$. Denoting $u:=-1 / z^{2}$, we have

$$
\begin{aligned}
2 u \alpha f\left(u / \mathrm{q}^{2}\right)= & -z^{-2} \alpha h(z \mathrm{q}) g(z \mathbf{q})+(z \mapsto-z) \\
= & h(z) z^{-3}\left(g(z / \mathbf{q})+z\left(z^{2}+E\right) g(z)\right)+(z \mapsto-z) \\
= & \left(-h(z / \mathbf{q})(\alpha z / \mathbf{q})^{-1} z^{-3} g(z / \mathbf{q})\right. \\
& +(z \mapsto-z))+\left(h(z)\left(1+E / z^{2}\right) g(z)+(z \mapsto-z)\right) \\
= & -u^{2} \mathbf{q} \alpha^{-1} 2 f\left(u \mathbf{q}^{2}\right)+(1-E u) 2 f(u) .
\end{aligned}
$$

Thus, $f \in V_{\mathrm{q}, \alpha, E}$.

Assuming $\omega(g) \neq 0$, we solve the equality $f=A_{g}(h)$ for $h$ as follows:

$$
\begin{aligned}
& 2 f(u) g(z \mathbf{q})+z \alpha g(z) 2 f\left(u / \mathbf{q}^{2}\right) \\
& \quad=(h(z)+z \alpha h(z \mathbf{q})) g(z) g(z \mathbf{q})+h(-z) g(-z) g(z \mathbf{q})+z \alpha g(z) h(-z \mathbf{q}) g(-z \mathbf{q}) \\
& \quad=h(-z)(g(-z) g(z \mathbf{q})+g(z) g(-z \mathbf{q}))=h(-z) \omega(g) .
\end{aligned}
$$

Thus, $h$ is determined through $f$ :

$$
h(z)=\left(f\left(-1 / z^{2}\right) g(-z \mathbf{q})-z \alpha g(-z) f\left(-1 /(z \mathbf{q})^{2}\right)\right) 2 / \omega(g) .
$$

Corollary 1. For any $\alpha \in \mathbb{C}_{\neq 0}$ and $E \in \mathbb{C}$, one has

$$
\operatorname{dim}\left(V_{\mathbf{q}, \alpha, E}\right)=\operatorname{dim}\left(T_{\mathbf{q},-\alpha}^{1}\right)=1 .
$$

In particular, the function

$$
\chi_{\mathbf{q}, \alpha, E}(z):=\vartheta(\alpha z ; \mathbf{q}) \psi_{\mathbf{q}, E}(z)+(z \mapsto-z)
$$

determines a basis element in $V_{\mathrm{q}, \alpha, E}$.

Proof. Indeed, $\psi_{\mathbf{q}, E}$ is an element of $G_{\mathbf{q}, E}$ with $\omega\left(\psi_{\mathbf{q}, E}\right)=2 \neq 0$, while $\vartheta(\alpha z ; \mathbf{q})$ determines a basis element in $T_{\mathrm{q},-\alpha}^{1}$.

Remark 8. In the proof of the second part of Proposition 2, we implicitly used an extension of the Wronskian pairing

$$
\begin{aligned}
& {[\cdot, \cdot]: G_{\mathbf{q}, E} \times V_{\mathbf{q}, \alpha, E} \rightarrow T_{\mathbf{q}, \alpha}^{1},} \\
& {[g, f](z)=g(z \mathbf{q}) f\left(\frac{-1}{z^{2}}\right)+z \alpha f\left(\frac{-1}{z^{2} \mathbf{q}^{2}}\right) g(z)}
\end{aligned}
$$

and the identity

$$
\left[g, A_{g}(h)\right](z)=h(-z) \omega(g) / 2, \quad \forall(g, h, z) \in G_{\mathbf{q}, E} \times T_{\mathbf{q},-\alpha}^{1} \times \mathbb{C}_{\neq 0} .
$$


Proposition 3. The multiplication of functions induces a linear map

$$
V_{\mathrm{q}, \alpha, E} \otimes T_{\mathrm{q}^{2}, \mathrm{q}^{2} \alpha}^{1} \rightarrow F_{\mathrm{q}, E}
$$

Proof. Let $f \in V_{\mathbf{q}, \alpha, E}, g \in T_{\mathbf{q}^{2}, \mathbf{q}^{2} \alpha}^{1}$ and $h:=f g$. We have

$$
\begin{aligned}
h\left(z / \mathrm{q}^{2}\right) & =(1-E z) f(z) g(z)-z^{2} \mathbf{q} \alpha^{-1} f\left(z \mathbf{q}^{2}\right) g(z) \\
& =(1-E z) h(z)-(z \mathbf{q})^{3} h\left(z \mathbf{q}^{2}\right) .
\end{aligned}
$$

Thus, $h \in F_{\mathrm{q}, E}$.

\subsection{Adjoint Functions Revisited}

Let $f \in V_{\mathrm{q}, \alpha, E}, g \in T_{\mathrm{q}^{2}, \mathrm{q}^{2} \alpha}^{1}$. Then, the adjoint function of the product $f g$ takes the form

$$
\widetilde{f g}(z)=\frac{f(z) g(z)}{\left[\phi_{\mathrm{q}, E}, f g\right](z)}=\frac{f(z)}{\left[\phi_{\mathrm{q}, E}, f\right](z)},
$$

where, in the last expression, by abuse of notation, we extend the Wronskian pairing to include the space $V_{\mathrm{q}, \alpha, E}$

$$
[\cdot, \cdot]: F_{\mathrm{q}, E} \times V_{\mathrm{q}, \alpha, E} \rightarrow T_{\mathrm{q}^{2}, \mathrm{q} / \alpha}^{2}, \quad[e, f](z)=e\left(\frac{z}{\mathrm{q}^{2}}\right) f(z)-\alpha z f\left(\frac{z}{\mathrm{q}^{2}}\right) e(z) \cdot(11
$$

The inclusions of vector spaces in (33) specified to $T_{\mathrm{q}^{2}, \mathrm{q} / \alpha}^{2}$ become

$$
T_{\mathbf{q}^{2}, \mathrm{q} / \alpha}^{2} \subset T_{\mathrm{q}^{2 n}, \mathbf{q}^{n^{2} / \alpha^{n}}}^{2 n}, \quad \forall n \in \mathbb{Z},
$$

which imply that

$$
\left[\phi_{\mathbf{q}, E}, f\right](z)=0 \Rightarrow\left[\phi_{\mathbf{q}, E}, f\right]\left(z \mathbf{q}^{2 n}\right)=0, \quad \forall n \in \mathbb{Z},
$$

and one has the equalities

$$
\check{f}\left(z \mathbf{q}^{2 n}\right)=\mathbf{q}^{n^{2}}(\alpha \mathbf{q} z)^{n} \check{f}(z), \quad \forall(n, z) \in \mathbb{Z} \times\left[\phi_{\mathbf{q}, E}, f\right]^{-1}(0) \cap U\left(\phi_{\mathbf{q}, E}\right),
$$

where

$$
\check{f}:=f / \phi_{\mathrm{q}, E} .
$$

By adjusting the normalisation of $f$, we can write an equality

$$
\left[\phi_{\mathbf{q}, E}, f\right](z)=\vartheta\left(z / s ; \mathbf{q}^{2}\right) \vartheta\left(z s \mathbf{q} / \alpha ; \mathbf{q}^{2}\right), \quad \forall z \in \mathbb{C}_{\neq 0},
$$

where $s=s(\alpha, \mathbf{q}, E)$ is a fixed zero of $\left[\phi_{\mathbf{q}, E}, f\right]$. We conclude that

$$
\left[\phi_{\mathbf{q}, E}, f\right](z)=0 \Leftrightarrow\left[\phi_{\mathbf{q}, E}, f\right]\left(\frac{\alpha}{\mathbf{q} z}\right)=0 .
$$

\section{Solution of the Schrödinger Equations}

Define the notation

$$
p_{x}:=e^{-2 \pi \mathrm{b} x}, \quad \bar{p}_{x}:=e^{-2 \pi \overline{\mathrm{b}} x} .
$$


Theorem 2. Let $(\zeta, \sigma, E, \bar{E}) \in \mathbb{C}^{4}$ be such that

$$
\check{f}\left(p_{\sigma}\right)=\mathbf{q} p_{\zeta+\sigma} \check{f}\left(\frac{p_{\sigma}}{\mathbf{q}^{2}}\right), \quad \check{\bar{f}}\left(\bar{p}_{\sigma}\right)=\overline{\mathrm{q}} \bar{p}_{\zeta+\sigma} \check{\check{f}}\left(\frac{\bar{p}_{\sigma}}{\overline{\mathbf{q}}^{2}}\right), \quad \Xi(\sigma)=\Xi(\zeta-\sigma),
$$

where

$$
f \in V_{\mathrm{q}, \mathrm{q} p_{\zeta}, E} \backslash\{0\}, \quad \bar{f} \in V_{\overline{\mathrm{q}}, \overline{\mathrm{q}} \bar{p}_{\zeta}, \bar{E}} \backslash\{0\}, \quad \check{f}:=\frac{f}{\phi_{\mathrm{q}, E}}, \quad \check{\bar{f}}:=\frac{\bar{f}}{\phi_{\overline{\mathrm{q}}, \bar{E}}}
$$

and

$$
\Xi(t):=-e^{\pi i t(t+2 \zeta)} \frac{\check{f}\left(p_{t}\right) \bar{p}_{t}}{\check{f}\left(\bar{p}_{t}\right) p_{t}} .
$$

Then, the function

$$
\Psi(x)=e^{2 \pi i c_{\mathrm{b}} x} \frac{e^{\pi i x^{2}} f\left(p_{x}\right) \phi_{\overline{\mathrm{q}}, \bar{E}}\left(\bar{p}_{x}\right)+\frac{p_{x}}{\bar{p}_{x}} \Xi(\sigma) e^{-2 \pi i \zeta x} \bar{f}\left(\bar{p}_{x}\right) \phi_{\mathrm{q}, E}\left(p_{x}\right)}{\vartheta\left(p_{x-\sigma} ; \mathrm{q}^{2}\right) \vartheta\left(p_{x+\sigma-\zeta} ; \mathrm{q}^{2}\right)}
$$

is a holomorphic solution of the system of difference Eqs. (55), (56).

Proof. Based on two possible asymptotics at $x \rightarrow+\infty$, the most general ansatz for the common eigenfunction of our spectral problem is of the form

$$
\Psi(x)=\Psi_{1}(x)+\Xi^{\prime} \Psi_{2}(x),
$$

where

$$
\begin{array}{ll}
\Psi_{1}(x):=\psi_{1}(x) \tilde{h}\left(p_{x}\right) \phi_{\overline{\mathbf{q}}, \bar{E}}\left(\bar{p}_{x}\right), & h \in F_{\mathbf{q}, E} \backslash \mathbb{C} \phi_{\mathbf{q}, E}, \\
\Psi_{2}(x):=\psi_{2}(x) \phi_{\mathbf{q}, E}\left(p_{x}\right) \tilde{\bar{h}}\left(\bar{p}_{x}\right), & \bar{h} \in F_{\overline{\mathrm{q}}, \bar{E}} \backslash \mathbb{C} \phi_{\overline{\mathbf{q}}, \bar{E}},
\end{array}
$$

and $\Xi^{\prime} \in \mathbb{C}$.

Remark 9. If the bar operation is the complex conjugation, the two functions are related as follows

$$
\overline{\Psi_{1}(x)}=e^{\pi i x^{2}} \Psi_{2}(x), \quad \forall x \in \mathbb{R} .
$$

That implies that if $\left|\Xi^{\prime}\right|=1$, then

$$
\Xi^{\prime} \overline{\Psi(x)}=e^{\pi i x^{2}} \Psi(x), \quad \forall x \in \mathbb{R} .
$$

An important additional condition for this ansatz, to be called Requirement $(I)$, is that the functions $\Psi_{1}(x)$ and $\Psi_{2}(x)$ should share one and the same set of poles.

If one chooses nonzero $h=f g$ and $\bar{h}=\bar{f} \bar{g}$, where

$$
(f, g) \in V_{\mathbf{q}, \alpha, E} \times T_{\mathbf{q}^{2}, \mathbf{q}^{2} \alpha}^{1} \text { and }(\bar{f}, \bar{g}) \in V_{\overline{\mathbf{q}}, \bar{\alpha}, \bar{E}} \times T_{\overline{\mathbf{q}}^{2}, \overline{\mathbf{q}}^{2} \bar{\alpha}}^{1}
$$

for some $\alpha, \bar{\alpha} \in \mathbb{C}_{\neq 0}$, then the denominators simplify to $\left[\phi_{\mathrm{q}, E}, f\right]$ and $\left[\phi_{\overline{\mathbf{q}}, \bar{E}}, \bar{f}\right]$ [(see eq. (110)] to become elements of 2-dimensional vector spaces $T_{\mathrm{q}^{2}, \mathrm{q} / \alpha}^{2}$ and $T_{\overline{\mathrm{q}}^{2}, \overline{\mathrm{q}} / \bar{\alpha}}^{2}$, respectively. By adjusting the normalisations of $f$ and $\bar{f}$, we can assume that

$$
\begin{aligned}
& {\left[\phi_{\mathbf{q}, E}, f\right](z)=\vartheta\left(\frac{z}{s} ; \mathbf{q}^{2}\right) \vartheta\left(\frac{z s \mathbf{q}}{\alpha} ; \mathbf{q}^{2}\right),} \\
& {\left[\phi_{\overline{\mathbf{q}}, \bar{E}}, \bar{f}\right](z)=\vartheta\left(\frac{z}{\bar{s}} ; \overline{\mathbf{q}}^{2}\right) \vartheta\left(\frac{z \bar{s} \overline{\mathbf{q}}}{\bar{\alpha}} ; \overline{\mathbf{q}}^{2}\right),}
\end{aligned}
$$


where

$$
s=s(E), \quad \bar{s}=\bar{s}(\bar{E})
$$

are some chosen zeros of $\left[\phi_{\mathbf{q}, E}, f\right]$ and $\left[\phi_{\overline{\mathbf{q}}, \bar{E}}, \bar{f}\right]$, respectively.

Remark 10. By solving Eq. (130) for $E=E(s)$ and $\bar{E}=\bar{E}(\bar{s})$, one can think of $s$ and $\bar{s}$ as independent variables.

In order to fulfil Requirement(I), to rewrite $\left[\phi_{\mathrm{q}, E}, f\right](z)$ by using the substitutions

$$
z \mapsto p_{x}, \quad \alpha \mapsto \mathrm{q} p_{\zeta}, \quad s \mapsto p_{\sigma}
$$

and the modularity property $(27)$

$$
\begin{aligned}
& {\left[\phi_{\mathbf{q}, E}, f\right](z) \mapsto \vartheta\left(p_{x-\sigma} ; \mathrm{q}^{2}\right) \vartheta\left(p_{x+\sigma-\zeta} ; \mathrm{q}^{2}\right)} \\
& \quad=\vartheta\left(\bar{p}_{x-\sigma} ; \overline{\mathbf{q}}^{2}\right) \vartheta\left(\bar{p}_{x+\sigma-\zeta} ; \overline{\mathrm{q}}^{2}\right) e^{2 \pi i\left(\left(x-\sin \theta-\frac{1}{2} \zeta\right)^{2}+\left(\sigma-\frac{1}{2} \zeta\right)^{2}\right)} \frac{i}{\mathrm{~b}^{2}} .
\end{aligned}
$$

Thus, Requirement(I) is fulfilled if we substitute in $\left[\phi_{\overline{\mathbf{q}}, \bar{E}}, \bar{f}\right]$

$$
\bar{\alpha} \mapsto \overline{\mathrm{q}} \bar{p}_{\zeta}, \quad \bar{s} \mapsto \bar{p}_{\sigma} .
$$

In this way, the first two relations in (119) become equivalent to (130).

Putting everything together, we obtain

$$
\Psi(x)=e^{2 \pi i c_{\mathrm{b}} x} \frac{e^{\pi i x^{2}} f(z) \phi_{\overline{\mathrm{q}}, \bar{E}}(\bar{z})+\Xi e^{-2 \pi i(\zeta+2 \sin \theta) x} \bar{f}(\bar{z}) \phi_{\mathrm{q}, E}(z)}{\vartheta\left(\frac{z}{s} ; \mathrm{q}^{2}\right) \vartheta\left(\frac{z s \mathrm{q}}{\alpha} ; \mathrm{q}^{2}\right)}
$$

with substitutions (131), (133) and $\bar{z} \mapsto \bar{p}_{x}$, and the parameter

$$
\Xi:=\Xi^{\prime} i e^{2 \pi i\left((\sin \theta+\zeta / 2)^{2}+(\sigma-\zeta / 2)^{2}\right)-2 i \theta}
$$

that we choose by the condition of cancellation of the pole of $\Psi(x)$ at $x=\sigma$ with the result

$$
\Xi=\Xi(\sigma):=-e^{\pi i \sigma(\sigma+2 \zeta)} \frac{\check{f}(s) \bar{s}}{\check{\bar{f}}(\bar{s}) s}
$$

equivalent to (121) under the substitutions $s \mapsto p_{\sigma}$ and $\bar{s} \mapsto \bar{p}_{\sigma}$.

Remark 11. Equality (136) implies that all the poles of $\Psi(x)$ at $\sigma+i \mathrm{~b} m+i \mathrm{~b} n$, $m, n \in \mathbb{Z}$, are cancelled as well. The proof is based on the relations (114) and their complex conjugate counterparts.

Now, the last step in the proof is to fulfil Requirement(II) which consists of cancelling the remaining poles of $\Psi(x)$. Due to the equivalence (117) and Remark 11, Requirement(II) boils down to a single equation

$$
\Xi(\sigma)=\Xi(\zeta-\sigma) \text {. }
$$

which determines a discrete set of solutions for the variable $\sigma$, while the corresponding eigenvalues of the Hamiltonian are given through the implicit function $E=E(\sigma)$.

In conclusion, we would like to remark that the variable $\zeta$ in Theorem 2 is an auxiliary parameter whose role is unclear to us. We conjecture that the 
eigenvalues of the Hamiltonian as well as the eigenvectors are independent of $\zeta$. This is confirmed by numerical calculations.

\section{Acknowledgements}

We would like thank Vladimir Bazhanov, Vladimir Mangazeev, Marcos Mariño and Szabolc Zakany for valuable discussions. We also would like to thank the anonymous referee whose remarks were very helpful for improving the mathematical rigour of our presentation, especially the part that concerns the formal normality of the Hamiltonian. The work is partially supported by the Australian Research Council and Swiss National Science Foundation.

Funding Open access funding provided by University of Geneva.

Open Access. This article is licensed under a Creative Commons Attribution 4.0 International License, which permits use, sharing, adaptation, distribution and reproduction in any medium or format, as long as you give appropriate credit to the original author(s) and the source, provide a link to the Creative Commons licence, and indicate if changes were made. The images or other third party material in this article are included in the article's Creative Commons licence, unless indicated otherwise in a credit line to the material. If material is not included in the article's Creative Commons licence and your intended use is not permitted by statutory regulation or exceeds the permitted use, you will need to obtain permission directly from the copyright holder. To view a copy of this licence, visit http://creativecommons. org/licenses/by/4.0/.

Publisher's Note Springer Nature remains neutral with regard to jurisdictional claims in published maps and institutional affiliations.

\section{References}

[1] Aganagic, M., Cheng, M.C.N., Dijkgraaf, R., Krefl, D., Vafa, C.: Quantum geometry of refined topological strings. J. High Energy Phys. 11, 019 (2012)

[2] Aganagic, M., Dijkgraaf, R., Klemm, A., Mariño, M., Vafa, C.: Topological strings and integrable hierarchies. Comm. Math. Phys. 261(2), 451-516 (2006)

[3] Babelon, O., Kozlowski, K.K., Pasquier, V.: Baxter operator and Baxter equation for $q$-Toda and Toda 2 chains. Rev. Math. Phys. 30(6), 1840003 (2018)

[4] V. V. Bazhanov, S. L. Lukyanov, and A. B. Zamolodchikov. Spectral determinants for Schrödinger equation and Q-operators of conformal field theory. In: Proceedings of the Baxter Revolution in Mathematical Physics (Canberra, 2000), volume 102, pages 567-576, 2001

[5] Codesido, S., Grassi, A., Mariño, M.: Spectral theory and mirror curves of higher genus. Ann. Henri Poincaré 18(2), 559-622 (2017)

[6] Dorey, P., Tateo, R.: Anharmonic oscillators, the thermodynamic Bethe ansatz and nonlinear integral equations. J. Phys. A 32(38), L419-L425 (1999)

[7] Faddeev, L.D.: Discrete Heisenberg-Weyl group and modular group. Lett. Math. Phys. 34(3), 249-254 (1995) 
[8] Grassi, A., Hatsuda, Y., Mariño, M.: Topological strings from quantum mechanics. Ann. Henri Poincaré 17(11), 3177-3235 (2016)

[9] Grassi, A., and Mariño, M. .: The complex side of the TS/ST correspondence. arXiv:1708.08642, (2017)

[10] Hatsuda, Y., Mariño, M., Moriyama, S., Okuyama, K.: Non-perturbative effects and the refined topological string. J. High Energy Phys. 9, 168 (2014)

[11] Hatsuda, Y., Moriyama, S., Okuyama, K.: Instanton effects in ABJM theory from Fermi gas approach. J. High Energy Phys. 1, 158 (2013)

[12] Huang, M.-X., Wang, X.-f, : Topological strings and quantum spectral problems. J. High Energy Phys. 9, 150 (2014)

[13] Källén, J., Mariño, M.: Instanton effects and quantum spectral curves. Ann. Henri Poincaré 17(5), 1037-1074 (2016)

[14] Kashaev, R.M., and Sergeev, S.M.: Spectral equations for the modular oscillator. Rev. Math. Phys., 30(7):1840009, 28, (2018). Preprint arXiv:1703.06016

[15] Kashani-Poor, A.-K.: Quantization condition from exact WKB for difference equations. arXiv:1604.01690, (2016)

[16] Mariño, M.: Spectral theory and mirror symmetry. In: String-Math 2016 of Proc. Sympos. Pure Math, vol. 98, pp. 259-294. Amer. Math. Soc., Providence, RI, (2018)

[17] Mariño, M., Putrov, P.: ABJM theory as a Fermi gas. J. Stat. Mech. Theory Exp. 3, P03001 (2012)

[18] Mariño, M., and Zakany, S.: Exact eigenfunctions and the open topological string. arXiv:1606.05297, (2016)

[19] Mironov, A., Morosov, A.: Nekrasov functions and exact Bohr-Sommerfeld integrals. J. High Energy Phys. 4, 040 (2010)

[20] Nekrasov, N.A., and Shatashvili, S.L.: Quantization of integrable systems and four dimensional gauge theories. In: XVIth International Congress on Mathematical Physics, pp. 265-289. World Sci. Publ., Hackensack, NJ, (2010)

[21] Schmüdgen, K.: Unbounded self-adjoint operators on Hilbert space. Grad-uate Texts in Mathematics, vol. 265. Springer, Dordrecht (2012)

[22] Sergeev, S.M.: A quantization scheme for modular $q$-difference equations. Teoret. Mat. Fiz. 142(3), 500-509 (2005) 
Rinat Kashaev

Section de Mathématiques

Université de Genève

2-4 rue du Lièvre, Case Postale 64

1211 Genève 4

Switzerland

e-mail: rinat.kashaev@unige.ch

\section{Sergey Sergeev}

Department of Theoretical Physics, Research School of Physics and Engineering Australian National University

Canberra

ACT 0200

Australia

e-mail: sergey.m.sergeev@gmail.com

Communicated by Jan Derezinski.

Received: May 3, 2019.

Accepted: September 12, 2020. 